\title{
Conformational Change and Orientation Fluctuations of Isotactic Polystyrene Prior to Crystallization
}

\author{
Go Matsuba, Keisuke KaJI, ${ }^{\dagger}$ Koji Nishida, Toshiji Kanaya, \\ and Masayuki IMAI* \\ Institute for Chemical Research, Kyoto University, Uji, Kyoto, 611-0011, Japan \\ * Department of Physics, Faculty of Science, Ochanomizu University, \\ Otsuka, Bunkyo-ku, Tokyo 112-0012, Japan
}

(Received February 23, 1999)

\begin{abstract}
In order to clarify the preparation process of crystal nucleation of polymers, we have studied the conformational changes and orientation fluctuations of isotactic polystyrene (iPS) in the induction period of crystallization using time-resolved Fourier transform infrared (FT-IR) spectroscopy and depolarized light scattering (DPLS) techniques, respectively. Their measurements were carried out for the so-called cold crystallization, when it was crystallized somewhat above the glass transition temperature $T_{\mathrm{g}}=100^{\circ} \mathrm{C}$ from the glassy states. It is found from the time-resolved FT-IR measurements that the polymer chains begin to assume alternate conformations of trans $(\mathrm{T})$ and gauche $(\mathrm{G})$ in the induction period, which are characteristic to the $3 / 1$ helix in the crystalline state. On the other hand, the time-resolved DPLS observations reveal that orientation fluctuations begin to grow in the induction period after a temperature jump to the crystallization temperature, which means that some rod-like segments of polymer chains actually start to orient parallel to one another prior to crystallization. These results are discussed in terms of the Doi's theory on the isotropic-to-nematic transition of liquid crystals where we assumed that the $3 / 1$ helical segments are stiff and have a persistence length being almost equal to the length of the heilx axis. Thus, the growth of the helices involves the increase in persistence length and hence in excluded volume, to cause the parallel ordering of the helix parts as stiff segments.

KEY WORDS Cold Crystallization / Isotactic Polystyrene / Induction Period / Conformational Change Parallel Orientation /
\end{abstract}

Recently, the structure formation during the induction period prior to crystal nucleation has received much attention. ${ }^{1-8}$ We investigated the crystallization processes of poly(ethylene terephthalate) (PET) when it was annealed just above the glass transition temperature $\left(T_{\mathrm{g}}\right)$ using small-angle X-ray scattering (SAXS), ${ }^{4,5}$ smallangle neutron scattering (SANS) ${ }^{6,7}$ and depolarized light scattering (DPLS) ${ }^{6.8}$ techniques. Surprisingly, we have discovered that a new SAXS peak following the kinetics of spinodal decomposition (SD) appears from the very early stage of the induction period prior to crystallization. ${ }^{4,5}$ Such SD can be understood based on the kinetic theory for the isotropic-to-nematic transition of liquid crystals presented by Doi et al. ${ }^{9-12}$; the parallel orientation of stiff polymer segments induces a SD type microphase separation with a characteristic wavelength being larger than the value corresponding to the SAXS peak. $^{4,5}$ In fact, the parallel orientation was experimentally observed by DPLS measurements. ${ }^{6,8}$ According to this theory, parallel ordering of rod-like polymers is induced only when their length exceeds a critical value. The SANS measurements with deuterium labeling ${ }^{6,7}$ revealed that the persistence length of PET chains, i.e. the length of stiff segments, actually increases in the induction period to exceed the critical value.

In the preceding paper, ${ }^{13}$ we investigated the crystallization processes of syndiotactic polystyrene with an extended chain conformation in the crystalline state by annealing at $120^{\circ} \mathrm{C}$, i.e., $20^{\circ} \mathrm{C}$ above $T_{\mathrm{g}}$ by means of timeresolved FT-IR and DPLS techniques. The FT-IR measurements have revealed that the rod-like segments in polymer chains extend as gauche conformations trans-

\footnotetext{
${ }^{\dagger}$ To whom correspondence should be addressed.
}

fer to trans ones during the induction period before crystallization. It was expected from the Doi's theory ${ }^{9-12}$ that this chain extension causes orientation fluctuations of the stiff segments when their length exceeds a critical value. Such orientation ordering in the induction period was actually confirmed by DPLS measurements.

It is interesting to investigate how the chain conformation changes during the induction period in the case of polymers assuming helical structure in the crystalline state. In this study, we have employed isotactic polystyrene (iPS) with $3 / 1$ helix as an example of helical polymers and investigated the structural changes during the induction period when the polymer was crystallized from the glassy state. The conformational changes were detected by a time-resolved FT-IR spectroscopic method. In this case, it is absolutely necessary that the IR bands concerned should be well characterized. Fortunately, as for isotactic polystyrene, they have been well characterized in terms of tetrad sequences of trans and gauche by Kobayashi et al. ${ }^{14,15}$ The parallel orientation of stiff segments during the induction period has also been measured for iPS by DPLS, and on the basis of these observed results, we discuss the structural formation processes during the induction period of crystallization, focusing on the relation between the $3 / 1$ helix formation and the parallel ordering of $3 / 1$ helix segments.

\section{EXPERIMENTAL}

The sample used for this study was isotactic polystyrene (iPS) with a weight-average molecular weight, $M_{w}=4.0 \times 10^{5}$ and a polydispersity, $M_{w} / M_{n}=2$. The glass transition temperature, $T_{\mathrm{g}}$, was determined by DSC measurements to be $100^{\circ} \mathrm{C}$. Amorphous thin films about 


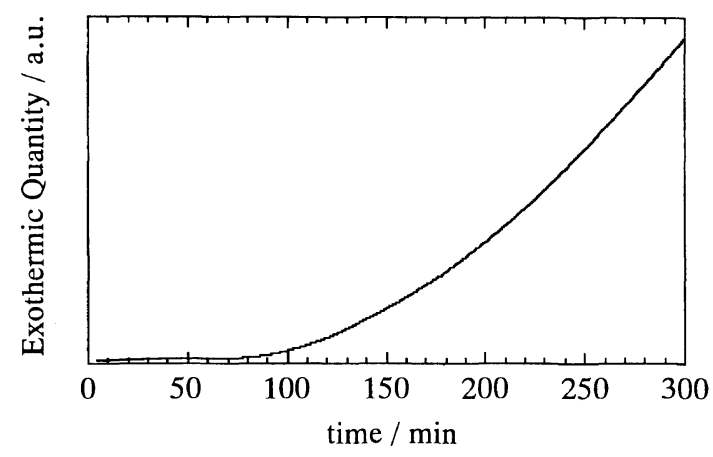

Figure 1. Annealing time dependence of crystallization isotherm for iPS glass. Annealing temperature was $135^{\circ} \mathrm{C}$.

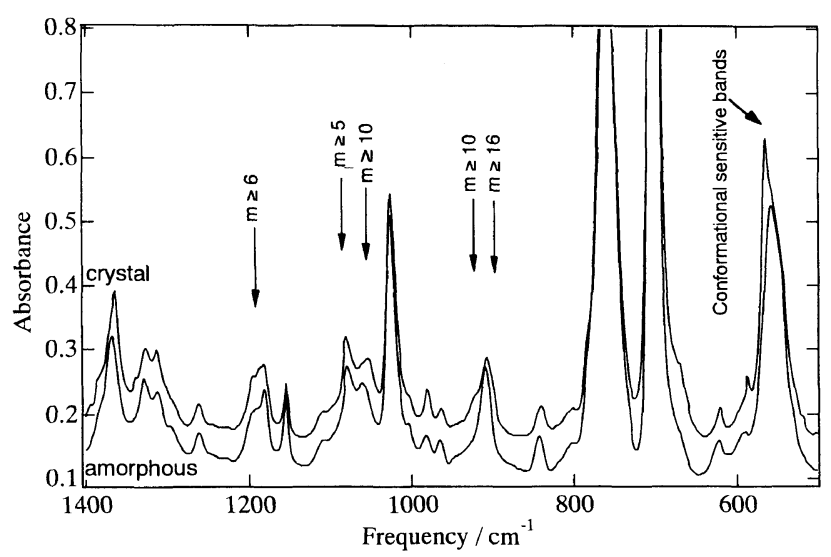

Figure 2. FT-IR spectra of crystalline (upper) and amorphous (lower) iPS in the region $500-1400 \mathrm{~cm}^{-1} . m$ indicates the minimum number of monomer units including in the $3 / 1$ helix parts of the polymer chain.

$50 \mu \mathrm{m}$ thick were obtained by quenching the iPS melt into ice-water after being hot-pressed at $290^{\circ} \mathrm{C}$ for $5 \mathrm{~min}$.

The time-resolved FT-IR measurements were performed on the melt-quenched sample under an isothermal condition at $135^{\circ} \mathrm{C}$, i.e., $35^{\circ} \mathrm{C}$ above $T_{\mathrm{g}}$ in a home-made temperature controlled cell. The IR absorption was recorded using a Nicolet Impact 410 system at $3.0 \mathrm{~min}$ intervals.

The time-resolved DPLS measurements were also carried out under the same annealing conditions at $135^{\circ} \mathrm{C}$. The samples were irradiated by a plane-polarized $\mathrm{He}-\mathrm{Ne}$ laser beam $(\lambda=632.8 \mathrm{~nm})$ on a hot-stage and the scattered light intensity under depolarized conditions was recorded by a photodiode array system at $0.5 \mathrm{~min}$ intervals.

\section{RESULTS AND DISCUSSION}

\section{Induction Period}

Figure 1 shows the crystallization isotherm $\phi(t)$ of iPS measured by differential scanning calorimetry (DSC) using Perkin Elmer DSC 7 as a function of annealing time at $135^{\circ} \mathrm{C}$. During the first $70 \mathrm{~min}$, neither exotherm nor endotherm is observed, indicating that it is in the so-called induction period. We also confirmed from wide-angle $\mathrm{X}$-ray diffraction measurements that no crystalline Bragg peaks except for amorphous halos are observed during the induction period; the measurements were carried out on several sample films quenched after annealing for given times before crystallization.

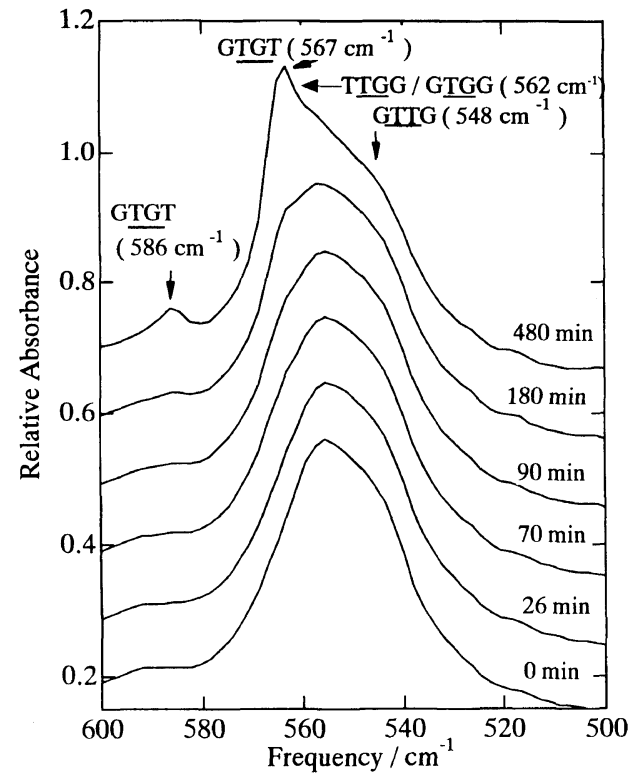

Figure 3. Annealing time dependence of IR spectra of iPS in the region $500-600 \mathrm{~cm}^{-1}$. The bands at $548,562,567$, and $586 \mathrm{~cm}^{-1}$ are assigned to GTTG, TTGG/GGTG, GTGT, and GTGT, respectively.

\section{Detection of Conformational Change by FT-IR}

Figure 2 shows the FT-IR spectra of iPS film samples melt-quenched and crystallized at $135^{\circ} \mathrm{C}$ for $400 \mathrm{~min}$ where the resulting crystal modification was the $\alpha$ form. ${ }^{16}$ Characteristic differences between the spectra of the amorphous and crystalline iPS films are seen on the bands at around $550 \mathrm{~cm}^{-1}$ which are sensitive to the chain conformation with tetrad sequences of trans $(\mathrm{T})$ and gauche $(\mathrm{G})^{15}$ and on the bands between 900 and 1300 $\mathrm{cm}^{-1}$ which are sensitive to the length of $3 / 1$ helix conformation. $^{14}$

Nakaoki and Kobayashi ${ }^{15}$ have assigned the absorption bands of polystyrenes at around $550 \mathrm{~cm}^{-1}$ to outof-plane modes of the phenyl group, whose frequencies depend on the local skeletal conformation in the neighborhood of the phenyl group. These bands are observed in both amorphous and crystalline spectra of iPS as seen in Figure 2 relating to the skeletal conformations with tetrad sequences as will be shown below.

In order to follow the conformational change in the induction period, we have carried out time-resolved FT-IR measurements after a temperature jump to $135^{\circ} \mathrm{C}$. Figure 3 shows the time evolution of the enlarged spectra in the frequency range 500 to $600 \mathrm{~cm}^{-1}$ when the sample was annealed at $135^{\circ} \mathrm{C}$. Here each curve is shifted along the axis of relative absorbance to make it easy to see. Four bands at 548,562, 567, and $586 \mathrm{~cm}^{-1}$ are distinguished and indicated with arrows in Figure 3: the $548 \mathrm{~cm}^{-1}$ band has been assigned to GTTG conformation, the $562 \mathrm{~cm}^{-1}$ band to TTGG and GGTG, and the 567 and $586 \mathrm{~cm}^{-1}$ bands to GTGT, respectively. Here T and $\mathrm{G}$ are trans and gauche conformations, respectively, and the two middle-underlined letters indicate the conformations around the $\mathrm{C}-\mathrm{C}$ bonds on the both sides of the carbon atom bonded to a phenyl group. For the quantitative analysis, we decomposed the spectra in the region $500-600 \mathrm{~cm}^{-1}$ into these four components by assuming a Lorentzian shape for each band as shown in 


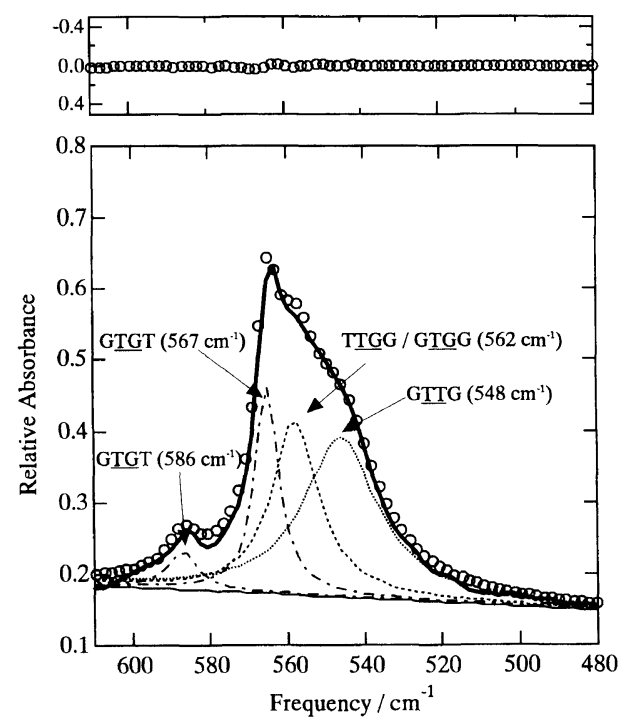

Figure 4. Separation of bands in the region $500-600 \mathrm{~cm}^{-1}$ of FT-IR spectrum of iPS. An example for the sample annealed at $135^{\circ} \mathrm{C}$ for $480 \mathrm{~min}$.

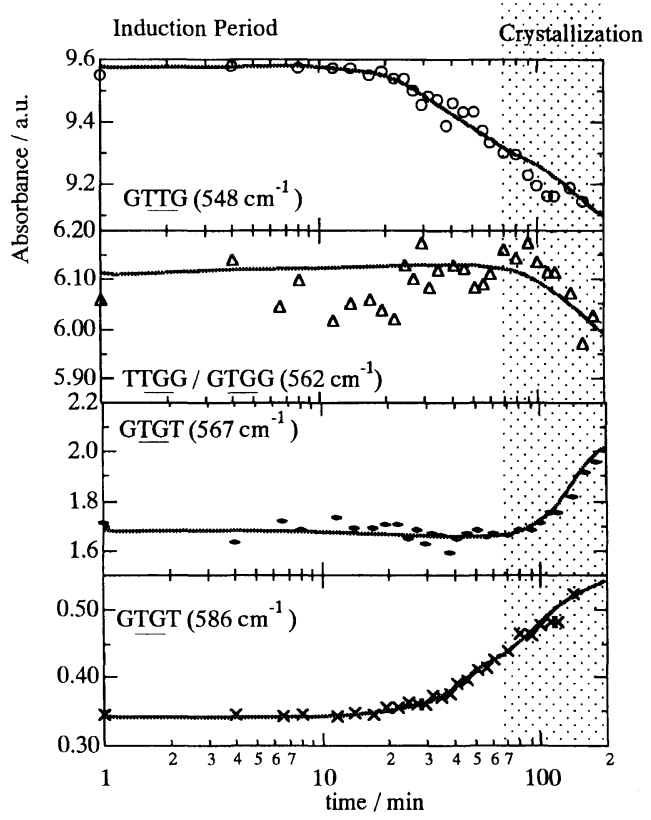

Figure 5. Annealing time dependence of the intensities of 548 (GTTG), 562 (TTGG/GGTG), 567 (GTGT), and 586 (GTGT) $\mathrm{cm}^{-1}$ The intensity is normalized to the value immediately after temperature jump to $135^{\circ} \mathrm{C}$.

Figure 4 for an example. The time evolution of intensities of these bands is plotted in Figure 5. If the polymer chain extends during the induction period, it is expected that GTGT bands increase in intensity because the conformation in the iPS crystal is the $3 / 1$ helix of $(\mathrm{TG})_{3}$. This expectation can qualitatively be confirmed in Figure 5; the peaks of GTGT (567 and $586 \mathrm{~cm}^{-1}$ ) increase in intensity with annealing time while those of GTTG $\left(548 \mathrm{~cm}^{-1}\right)$ and TTGG/GGTG $\left(562 \mathrm{~cm}^{-1}\right)$ decrease. The change of the $\overline{586} \mathrm{~cm}^{-1}$ band may imply that the formation of $3 / 1$ helix starts to occur and proceeds even during the induction period of crystallization. In the crystallization stage after the induction period, the bands of GTGT conformations continue to increase in intensity.

$\overline{\mathrm{On}}$ the other hand, several bands in the region

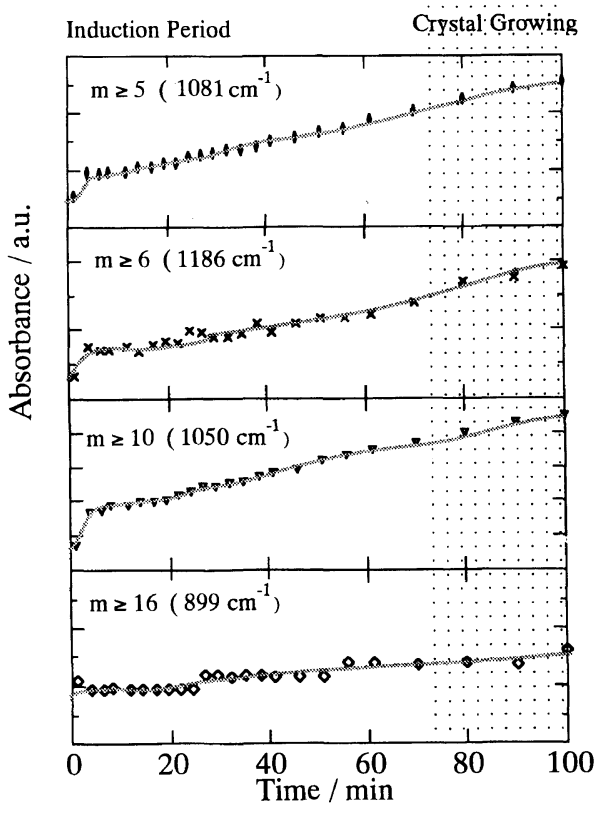

Figure 6. Annealing time dependence of FT-IR absorbance for the bands of $3 / 1$ helix containing $m$ or more monomers: $m \geq 5\left(1081 \mathrm{~cm}^{-1}\right)$, $m \geq 6\left(1186 \mathrm{~cm}^{-1}\right), m \geq 16\left(899 \mathrm{~cm}^{-1}\right)$ and $m \geq 10\left(1050 \mathrm{~cm}^{-1}\right)$. The intensity is normalized to the value immediately after temperature jump to $135^{\circ} \mathrm{C}$.

$900-1200 \mathrm{~cm}^{-1}$ of Figure 2 are assigned to $3 / 1$ helix bands by Kobayashi et al. ${ }^{14}$ Their frequencies depend largely on the sequence length of the helix part, i.e., the number $m$ of monomer units in the helix part. The band at $899 \mathrm{~cm}^{-1}$ is assigned to the $3 / 1$ helical segments including 16 or more monomer units $(m \geq 16)$, the both bands at 920 and $1050 \mathrm{~cm}^{-1}$ are assigned to the helical ones with 10 or more units $(m \geq 10)$, and the bands at 1081 and $1186 \mathrm{~cm}^{-1}$ to those with 5 or more $(m \geq 5)$ and 6 or more $(m \geq 6)$ units, respectively.

In order to see the growth of the $3 / 1$ helix bands during the induction period of crystallization, annealing time dependence of the intensities of the $3 / 1$ helix bands are shown in Figure 6 . The absorbance of the $3 / 1$ helix bands for $m \geq 5,6$ and 10 gradually increases in intensity after a slight abrupt increase in the induction period while that for $m \geq 16$ increases considerably late, indicating that naturally it takes more time for the longer helix $(m \geq 16)$ to be formed than the shorter ones $(m \leq 10)$. In any case, the present FT-IR results show that in the induction period the $3 / 1$ helix conformation increases and the polymer chains are extended with annealing time. It is also noted that the $3 / 1$ helix bands continue to increase in intensity even after the beginning of crystallization.

The abrupt increase in absorbance between 0 and $3.0 \mathrm{~min}$ for $m \geq 5,6$ and 10 as described above may mean the slight increase of the helical sequence length in the very early stage of the induction period. A similar initial extension of polymer chains was directly observed for PET by measuring persistence length using the SANS technique with a deuterium labeling method. ${ }^{6,7}$ Though the persistence length of iPS has not directly been measured yet, the increase in absorbance of $3 / 1$ helix bands may correspond to the increase of persistence length. The gradual increase between 3.0 and 70 min may indicate the gradual extension of polymer chains. 


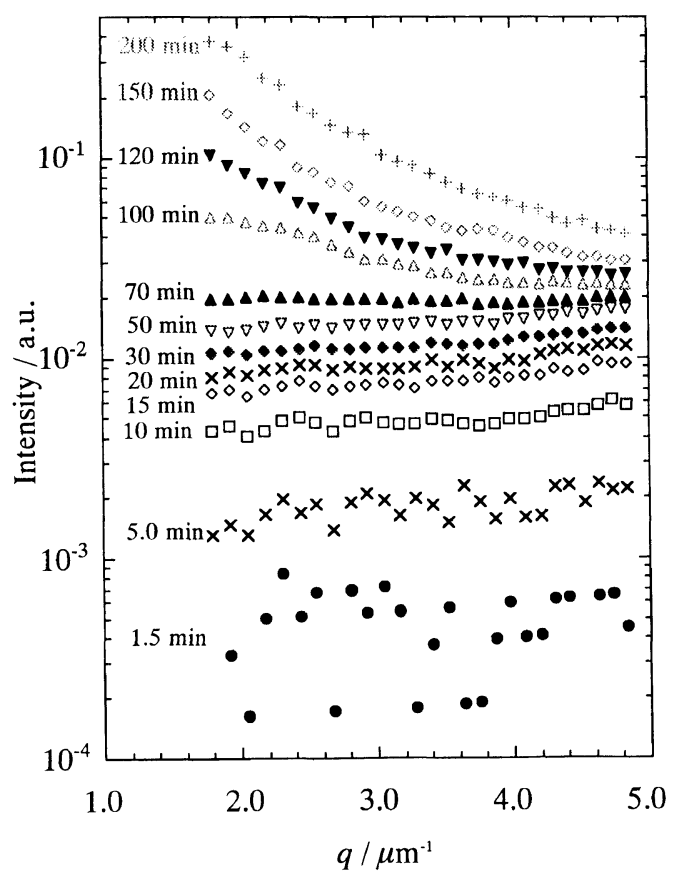

Figure 7. Depolarized light scattering profiles $I_{\mathrm{VH}}(q)$ of iPS films annealed at $135^{\circ} \mathrm{C}$ as a function of annealing time.

Anyway, the present results of time-resolved FT-IR measurements suggest that the growth of chain conformations of $3 / 1$ helix actually causes the chain extension during the indication period of crystallization.

\section{Orientation Fluctuations from DPLS Measurements}

As described in the Introduction, it is expected that the orientation fluctuations of stiff segments occur in the induction period when stiff segments extend over a critical value. In order to confirm whether or not such orientation fluctuations actually evolve with time during the induction period, DPLS measurements were performed under the same annealing conditions as for the FT-IR investigation. Figure 7 shows the semi-logarithmic expression of time evolution of the DPLS intensity at $135^{\circ} \mathrm{C}$ as a function of the length of scattering vector, $q$. There is no doubt that the depolarized intensity increases with the annealing time even in the induction period, suggesting that parallel ordering of polymer chain segments proceeds before crystallization. According to Stein et al., ${ }^{17}$ the Rayleigh factor $R_{+}(q)$ for depolarized light scattering from solids having randomly correlated orientation fluctuations can be expressed by

$$
R_{+}(q)=\left(\frac{\omega}{c}\right)^{4} \frac{\left\langle\delta^{2}\right\rangle}{15} \int_{0}^{\infty} g(r) \frac{\sin (q r)}{q r}(4 \pi r) \mathrm{d} r
$$

where $\omega$ is the angular frequency of incident radiation, $c$ is the velocity of light, $\left\langle\delta^{2}\right\rangle$ is the mean-square anisotropy and $g(r)$ is the function of orientation defined as $g(r)=\left(3\left\langle\cos ^{2} \phi_{i, j}\right\rangle_{\mathrm{r}}-1\right) / 2$, where $\phi_{i, j}$ is the angle between the optical axes of the $i$ th and $j$ th elements. The invariant due to the orientation fluctuations $I_{\mathrm{VH}}$ is given by

$$
I_{\mathrm{VH}}=\int_{0}^{\infty} R_{+}(q) q^{2} \mathrm{~d} q=\frac{2 \pi^{2}}{15}\left(\frac{\omega}{c}\right)^{4}\left\langle\delta^{2}\right\rangle
$$

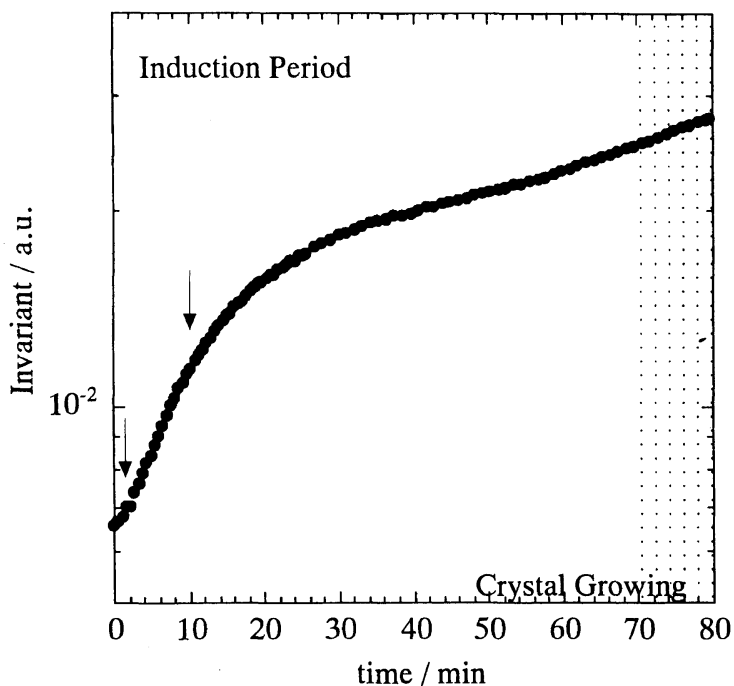

Figure 8. Annealing time dependence of the invariant for the depolarized light scattering intensity of iPS films in the induction period of crystallization.

These eq 1 and 2 give us a basis for interpretations of the results on the depolarized light scattering measurements.

As seen in Figure 7, during the induction period of crystallization the scattering profiles are almost independent of scattering vector, $q$, and the total intensity increases with annealing time. This result indicates that the sizes of oriented domains or their aggregates are much smaller than the scales corresponding to the measured $q$-range. But in the crystal growing period, the scattering profile becomes $q$-dependent; the intensity increases with decreasing $q$. This means that after the crystal growth the sizes of oriented domains or their aggregates have become comparable with the scale of $q$-range examined, which is probably because of the formation of spherulites. Similar results were observed in the crystallization processes of $\mathrm{PET}^{6,8}$ and SPS. ${ }^{13}$

Figure 8 shows the time dependence of the invariant for the orientation fluctuations $I_{\mathrm{VH}}$ (eq 2), calculated within the present $q$-range. In the very early stage until about $3.0 \mathrm{~min}$, the growth rate of the invariant is slow compared with that after $3.0 \mathrm{~min}$. In this time region, we observed the abrupt increase of IR bands originated from the $3 / 1$ helix conformation (see Figure 6). After this small increase, the invariant shows an exponential growth in the period between 3.0 and $10 \mathrm{~min}$. Such exponential growth is one of the characteristic features of spinodal decomposition (SD) in the kinetics of isotropic-nematic transitions of liquid crystals as shown by Doi et al., ${ }^{9-12}$ and was actually observed in the induction period of $\mathrm{PET}^{6,8}$ and $\mathrm{sPS}^{13}$ crystallization. Then we can conclude that the orientation fluctuations are caused by the parallel ordering of the $3 / 1$ helix segments in the induction period, following the kinetic process of isotropic-nematic transition. However, the initial delay until $3.0 \mathrm{~min}$ was not observed in the cases of PET and SPS, both of which assume extended conformations. Hence the time of the first $3.0 \mathrm{~min}$ after the temperature jump may be required for the helical segments to exceed a critical length. In the time range after $10 \mathrm{~min}$ the invariant shows a tendency of leveling-off while it begins to increase more steeply 
when the crystallization starts after $70 \mathrm{~min}$. It is natural to consider that the steeper increase is caused by the growth of spherulites.

Doi et al. have theoretically investigated dynamics of liquid crystalline phase formation in a solution of stiff polymers using a kinetics of two order parameters of concentration and orientation. This theory predicts that the transition from isotropic to nematic state occurs when the system is brought into a thermodynamically unstable region. This unstable state is caused by the increase of excluded volume due to the polymer chain extension. The critical concentration at which the isotropic phase becomes unstable is given by ${ }^{9}$

$$
v^{*}=\frac{4.19}{b L^{2}}
$$

where $b$ and $L$ are the diameter and the length of the rod-like polymer and the term $b L^{2}$ is in the order of the excluded volume of this polymer. When the mean number $v$ of the rod-like polymers per unit volume is smaller than $v^{*}$, the system is stable and the orientation fluctuations cannot grow with time, but when $v>v^{*}$, the system becomes unstable and the orientation fluctuations start to grow. This theory also predicts that the dynamics of isotropic-nematic transition is very similar to that of the spinodal-decomposition type phase separation. As shown above, we observed an exponential growth of orientation fluctuations between 3.0 and $10 \mathrm{~min}$ in the induction period (see Figure 8), which is one of the characteristic features of spinodal-decomposition type microphase separation. In what follows, we will examine whether the iPS system is under the unstable condition $\left(v>v^{*}\right)$ when the orientation fluctuations begin to occur.

Although this theory was originally formulated for the polymer solutions of stiff polymers, we found that it is applicable to a flexible sPS in the bulk system ${ }^{13}$ by assuming the hypothetical freely jointed chain model proposed by Flory $^{18}$ where the chain consists of connected stiff segments of a length being equal to its persistence length. Therefore, we again apply the theory to iPS that is also a flexible polymer. We have evaluated the criterion for the isotropic-nematic transition as follows. The concentration $v$ of rod-like segments in the glass can be evaluated from the persistence length $L$ of the polymer and the density $\rho$, using eq 4

$$
v=\frac{\rho N_{\mathrm{A}}}{\left(\frac{L}{l_{0}}\right) M_{0}}
$$

where $l_{0}$ and $M_{0}$ are the length and molecular weight of the monomer, respectively, and $N_{\mathrm{A}}$ is the Avogadro number. To describe the chain conformation of iPS the helical wormlike (HW) touched-bead model was employed. ${ }^{19,20}$ For the glassy state of iPS, the persistence length $L$ is given as $1.32 \mathrm{~nm}$, and the density measured by flotation method was $1.04 \mathrm{~g} \mathrm{~cm}^{-3}$. Further, $b$ and $l_{0}$ can be taken as $1.40 \mathrm{~nm}$ and $0.222 \mathrm{~nm}$, respectively. With these values, $v$ and $v^{*}$ are calculated to be 1.01 and 1.72 segments $\mathrm{nm}^{-3}$, respectively. In this condition $\left(v<v^{*}\right)$, the system is stable and the orientation fluctuations do not grow. In order to get into the unstable region, polymer chains must extend to reduce the critical concentration of eq 3 . After annealing, the conformational changes begin to occur and the persistence length may increase because of the growth of $3 / 1$ helix conformation. We assume that the persistence length is close to the average length of $3 / 1$ helix parts. Then the length, $L$, must increase with annealing time during the induction period, leading to the decrease of $v^{*}$ (see eq 3 ). When the average length of the rod-like segments exceeds $2.24 \mathrm{~nm}$, which gives a condition of $v=v^{*}$, the iPS system gets into the unstable region. From conformational point of view, $2.24 \mathrm{~nm}$ corresponds to ten monomers $(m=10)$ having five alternate trans and gauche conformations. This result seems to be reasonable because as seen from Figure 6, the bands for $m \leq 10$ start to increase in intensity from the very beginning but the intensity of the $m \geq 16$ band grows considerably late after about $25 \mathrm{~min}$ while the orientation occurs from the beginning (see Figure 8). The extension from 6 monomers to 10 monomers of the stiff segments requires a considerable time, which would cause the delay of $3 \mathrm{~min}$ in the increase of the DPLS invariant.

\section{CONCLUSIONS}

In order to elucidate the structure formation mechanism prior to crystal nucleation, we performed time-resolved FT-IR and depolarized light scattering measurements on iPS during the induction period of crystallization from the glassy state. In the time-resolved FT-IR measurements, the increase of alternating trans and gauche conformations is observed immediately after the temperature jump to $135^{\circ} \mathrm{C}$ as well as the increase of $3 / 1$ helix bands. Further, the time-resolved DPLS measurements revealed that the orientation fluctuations begin to increase in the induction period, indicating that parallel ordering of polymer chains actually proceeds in the induction period. However, the orientation fluctuations of the main chain begin to arise at $3.0 \mathrm{~min}$ after temperature jump. These results suggest that in the case of helical the critical length of stiff segments considerably large, resulting in the delay of the parallel ordering of polymer chains. This is contrast to the cases of PET and sPS. The critical length for iPS was tentatively determined from the Doi's theory on isotropic-nematic transition of liquid crystalline polymers.

Acknowledgment. This study has been supported by NEDO International Joint Research Grant for the project "Fundamental Studies on Crystallization of Polymers" and by the Grant-in-Aid for Scientific Research on Priority Area "Cooperative Phenomena in Complex Liquids" from the Ministry of Education, Science, Sports and Culture of Japan. The authors greatly appreciate these foundations.

\section{REFERENCES}

1. T. A. Ezquerra, E. López-Cabarcos, B. S. Hsiao, and F. J. Baltá Calleja, Phys. Rev. E, 54, 989 (1996).

2. T. Kimura, H. Ezure, S. Tanaka, and E. Ito, J. Polym. Sci. B, 36, 1227 (1998).

3. N. J. Terrill, P. A. Fairclough, E. Towns-Andrews, B. U. Komanschek, R. J. Young, and A. J. Ryan, Polym. Comm., 39, 2381 (1998). 
4. M. Imai, K. Mori, T. Mizukami, K. Kaji, and T. Kanaya, Polymer, 33, 4451 (1992).

5. M. Imai, K. Mori, T. Mizukami, K. Kaji, and T. Kanaya, Polymer, 33, 4457 (1992).

6. M. Imai, K. Kaji, T. Kanaya, and Y. Sakai., Phys. Rev., B52, 12696 (1995).

7. M. Imai, K. Kaji, T. Kanaya, and Y. Sakai., Physica B, 213\&214, 718 (1995)

8. M. Imai, K. Kaji, and T. Kanaya, Phys. Rev. Lett., 71, 4162 (1993).

9. M. Doi and S. F. Edwards, "The Theory of Polymer Dynamics," Oxford University Press, Oxford, 1986, Chapter 10.

10. T. Shimada, M. Doi, and K. Okano, J. Chem. Phys., 88, 2815 (1988).

11. M. Doi, T. Shimada, and K. Okano, J. Chem. Phys., 88, 4070 (1988).
12. T. Shimada, M. Doi, and K. Okano, J. Chem. Phys., 88, 7181 (1988).

13. G. Matsuba, K. Kaji, K. Nishida, T. Kanaya, and M. Imai, Macromolecules, submitted.

14. M. Kobayashi, K. Akita, and H. Tadokoro, Makromol. Chem., 118, 324 (1968)

15. T. Nakaoki and M. Kobayashi, J. Mol. Struct., 242, 315 (1991).

16. G. Natta, P. Corradini, and I. W. Bassi, Nuovo Simento, Suppl., 15, 68 (1960).

17. R. S. Stein and P. R. Wilson, J. Appl. Phys., 33, 1914 (1962).

18. P. J. Flory, "Statistical Mechanics of Chain Molecules," Interscience, New York, N. Y., 1969.

19. H. Yamakawa, Ann. Rev. Phys. Chem., 35, 23 (1984).

20. Y. Einaga, H. Koyama, T. Konishi, and H. Yamakawa, Macromolecules, 22, 3419 (1989). 